\title{
Energy Deficiency Leading to Predisposition of Formation of Malignant Ovary Tumor Secondary to Chronic Use of Hormones to Treat Acne
}

\author{
Huang WL* \\ Paulo, Brazil \\ *Corresponding author: Huang Wei Ling, MD, Rua Homero Pacheco Alves, 1929, Franca, São Paulo, 14400-010, Brazil
}

Infectious Diseases, General Practice, Medical Acupuncture and Pain Management, Medical Acupuncture and Pain Management Clinic, Franca, São

Received: October 06, 2021; Accepted: October 14, 2021; Published: November 24, 2021

\begin{abstract}
Introduction: Malignant ovary tumor is the one of highest mortality rate in gynecology cancer and the induction of this ovary cancer is related to metabolism and exposure to estrogen.

Purpose: To demonstrate that the use of hormones to treat acne is not safe, mainly in patient with energy deficiency in the chakras' energy centers and the possibility of inducing cancer in the ovaries could be one of the reasons to reduce the indication of these medications to treat acne.

Methods: One case report, 29-years-old women with pain in the abdomen and after doing one ultra-sound that revealed an alteration in the right ovary that was bigger than normal and the surgery was scheduled to see what could be.

Results: In the surgery, it was done biopsy that revealed malignant tumor in the right ovary. The patient needs to take out all the womb and part of the ovary due to malignance. The patient was submitted to chemotherapy, radiotherapy and started treatment with acupuncture with apex ear bloodletting and did the chakras' energy measurement that revealed that all the chakras' were in the lowest level of energy. Homeopathy was used according to the theory Constitutional Homeopathy of the Five Elements based on Traditional Chinese Medicine and crystal-based medications to replenish the energy of the chakras' energy centers. The patient said that she was taking hormones for ten years hormones to treat acne in the skin. Today, she still working at the local secretary and have to put a hair wig due to hair loss when using chemotherapy.

Conclusion: The chronic use of hormones can induce the formation of malignant ovary tumor especially when the patient has lack of energy of the chakras' energy centers diagnosed by radiesthesia procedure. The treatment of this lack of energy using Chinese dietary counseling, auricular acupuncture and homeopathies medications according to Constitutional Homeopathy of the Five Elements based in Traditional Chinese Medicine and crystalbased medications is the major importance to restore the energy of the patient that was low, even after the treatment using surgery, chemotherapy and radiotherapy. There is a need to prescribe very carefully hormones in the treatment of any pathology nowadays due to lack of energy of the entire population, leading to increase risks to formation of any type of cancer, in this case, the formation of malignant ovary tumor.
\end{abstract}

Keywords: Malignant ovary tumor, Hormones, Acne, Diet, Acupuncture, Traditional Chinese medicine, Chakras, Homeopathy.

\section{Introduction}

Cancer is the most common cause of mortality in most parts of the world, and currently is the most common impediment to achieving desirable life expectancy in most countries. Ovarian cancer is one of the most common gynecologic cancers that rank third after cervical and uterine cancer. It also has the worst prognosis and the highest mortality rate [1].

Although ovarian cancer has a lower prevalence in comparison with breast cancer, it is three times more lethal. The high mortality rate of ovarian cancer is caused by asymptomatic and secret growth of the tumor, delayed onset of symptoms, and lack of proper screening that result in it diagnosis in the advanced stages [1].

Like many cancers, the incidence of ovarian cancer varies across the world. The epidemiological diversity of ovarian cancer in different regions can be attributed to the risk factors that account for the occurrence of ovarian cancer. The highest prevalence of ovarian cancer is seen in non-Hispanic white women (12.0 per 100,000), followed by Hispanic (10.3 per 100,000), non-Hispanic black $(9.4$ per 100,000), and Asian/Pacific Islander women (9.2 per 100,000) [1].

According to Momenimovahed (2019), 295,414 cases of ovarian cancer have been identified in 2018, accounting for 3.4\% of all cancer cases in women. In 2018, 184,799 deaths occurred due to ovarian cancer, accounting for $4.4 \%$ of the entire cancer-related mortality among women [1].

\section{Purpose}

The purpose of this study is to demonstrate that patients with malignant ovary tumor have chakras' energy centers deficient in energy, predisposing them to have malignant cancer when using chronically hormones to treat acne. 


\section{Methods}

Through an extensive search for articles indexed in PubMed in Western medicine and in traditional Chinese medicine regarding malignant ovary cancer and in acne formation and through one case report of patient that had malignant ovary tumor formation after taking ten years of hormones to treat the acne.

The patient is a 29 -year-old woman that had a history of since the age of 18 years-old, was using oral contraceptive for the treatment of her face acnes. In June 2019, she went to a routine appointment but had some discomfort such as cramps spaced out and a lot of desire to go to the bathroom, she felt that her bladder was not emptying as it should, despite never having problems with colic, even before doing use of the contraceptive, which she used exclusively to treat her acne, correctly following the dosage of the medication, made only blood tests and Papanicolaou test, which apparently was all right.

In July 2019, nothing had changed and she was convinced that she had a urine infection and decided to go to the doctor on duty, so she did more tests and nothing was found, until she had a transvaginal ultrasound where it was detected that there was a pelvic mass and a lot of liquid in the abdominal cavity. She did more tests and came to the conclusion that surgery would be necessary, where they could give her a more accurate diagnosis. The doctor did the biopsy and she was diagnosed with malignant epithelial cancer of stage IIIC ovary. A total hysterectomy and administration of chemotherapy and also radiotherapy was required, where 6 cycles and maintenance therapy were performed.

During the chemotherapy sessions, she also went to my clinic, as her parents were also my patients. I measured her chakras' energy centers through radiesthesia procedure that revealed that all her five massive organs were without any energy (rated in one), only the spiritual chakra (seventh chakra) was normal (rated in eight) that was already expected, since the cause of cancer in TCM is energy deficiency and formation of internal Heat.

The first step in her treatment to improve her energy was to change her dietary habits according to Chinese dietary counseling, to do not imbalance even more the energy of the patient that was already very low. I recommended to her to avoid all dairy products, raw foods, sweets and cold drinks to do not imbalance the Spleenpancreas meridian that is responsible for the absorption of nutrients and production of Blood. I also suggest to the patient to do not drink soda, coffee and mate tea to do not imbalance even more the Kidney meridian, responsible for the production of Yin and Yang energy inside the body. Lastly, to do not eat fried foods, chocolate, honey, coconut, eggs, alcoholic beverages and melted cheese to do not imbalance the Liver and Gallbladder meridian because these foods can induce the formation of more internal Heat.

She also did some acupuncture session with me and I used auricular acupuncture associating apex ear bloodletting. The auricular acupuncture points used in her treatment were: Shen-men; Kidney, Liver, Spleen, Lungs, Heart, Large intestine, Occiput, Hunger point, endocrine. It was also used moxibustion therapy in some points in the belly and in the lumbar region, to increase the production of Yin and Yang energy.
And I also prescribe to her homeopathies medications according to the theory created by me (2020) entitled Constitutional Homeopathy of the Five Elements Based on Traditional Chinese Medicine. The use of highly diluted medication in this situation is very pertinent and I will explain in the discussion section why patients nowadays, need to use highly diluted medications instead of highly concentrated medications, in the discussion section.

Due to the COVID-19 pandemic, she suspended the acupuncture sessions for one year or more and in September 2020, she was discharged from the treatment where Blood and imaging tests were carried out to prove the remission of the disease.

However, in January 2021, when carrying out control tests, she did not have such satisfactory results, the disease had a relapse where it was necessary to return with chemotherapy treatment and she did not return again to continue her energy based treatment, probably due to the fear of COVID-19 infection, as she was considered immunosuppressed patient, due to cancer diagnosis.

According to the patient herself, the treatment with acupuncture and homeopathies helped a lot during the treatment with chemotherapy, not allowing her to have so many side effects and with a good mental health, making the results of the Western treatment better.

\section{Discussion}

This article will be written following Hippocrates (460 bce - 375 bce) oath that said that it is important to consider older ancient medical traditions prior to the knowledge we have nowadays [2].

So, to understand in the deeper sense what could be happening in this specific patient that was using chronically hormones to treat acne, for more than 10 years, it is important to understand what could be happening in this patient in the energy level, to establish the preventive measurements and not just treating the symptom itself, that is the ovary cancer formation [3].

Estrogens are implicated as causative factors of ovarian carcinogenesis Estrogens have long been suspected as etiologic factors of ovarian carcinoma. Although usage of estrogen-based oral contraceptives is known to reduce ovarian carcinoma risk, its effect is primarily attributed to reduction in ovulation frequency. Further wise literature from breast cancer research has demonstrated direct genotoxic effects of estrogen. Hence, it is logical to speculate that genomic damage of ovarian surface epithelium cells, covering the ovulating follicles or in inclusion cysts may, in part, be caused by the high levels of estrogen in the follicular fluid or in the ovarian stroma [4].

The first studies showed that the adult female acne lesions were located mainly on the lower part of the face, including the mandibular region, the perioral region and the chin, conferring a U-shape, in addition to the anterior cervical region. It is characterized by inflammatory lesions, papules and pustules, of mild to moderate intensity, with the presence of few closed comedowns or micro cysts. Post inflammatory hyperpigmentation is common and scars can occur in $20 \%$ of affected women. In addition, the skin may be more sensitive than that of adolescents, with less tolerance to topical medications [5]. 
Adult female acne is a therapeutic challenge because it presents a tendency to relapse, even after cycles of oral antibiotics or isotretinoin. The typical evolution of adult female acne, with frequent relapses, makes maintenance treatment essential [5].

But in this article, I will show a different point of view of carcinogenesis and briefly about acne, following what Hippocrates said that "Foolish the doctor who despites the knowledge acquired by the ancients", I will show how could be the formation of cancer induced by the chronic ingestion of hormones, according to the traditional Chinese medicine point of view [3].

The reasoning used to treat all my patients was based in one case I treated in 2006 and changed completely the way of thinking after this specific case, that I will describe now [6].

This patient was a 70 -year-old-male patient, who reported pain in the legs and was using anti-inflammatory medications for about 6 months without any improvement. He was diagnosed with KidneyYang deficiency, according to TCM. He received treatment with Chinese dietary counseling, acupuncture and auricular acupuncture associated with apex ear bloodletting [6].

With the treatment done, the pain in the legs diminished and the patient was submitted to an interview after 30 days of the treatment. The patient revealed that his eye pressure had also lowered from 40 $\mathrm{mmHg}$ to $17 \mathrm{mmHg}$ with the treatment he received for his leg pain, as his ophthalmologist confirmed. During the treatment, he had not reported to be treating glaucoma in the last 40 years with no improvement of his condition [6].

This unusual case became the cornerstone of all my studies in the field, trying to comprehend how the treatment focused on the root of the problem could treat different diseases and symptoms simultaneously and using the same method [7].

For this, I need to show some concepts and theories of TCM, for a better comprehension. I will use this tree like figure that is a metaphor of what level Western and a traditional Chinese medicine is treating nowadays, to us to know what we need to do. Western medicine looks to the part above the ground, with the branches and leaves; each branch represents one medical specialty and the leaves of this branch are the symptoms and diseases related to each specialty. So, in this case, the acne is one leaf of the dermatology branch and the ovarian tumor is one leaf of the gynecology branch. For the other hand, TCM looks to the entire tree, including the root that is what is nourishing and maintaining the health of the entire tree, and also, receiving the influences of the external pathogenic factors, which can begin the process of formation of disease, according to traditional Chinese medicine Figure 1 [7].

On the root of the tree, there are two theories in traditional Chinese medicine, that are Yin and Yang theory and the Five Elements theory. Yin and Yang are the two opposite forces that are believed to be present in all phenomena in the world. According to TCM, Yin and Yang are composed by four aspects: they are opposites; they are interdependent; they are mutually transformative, one always transforming into the other in a cyclical way; they are mutually consuming as well, because one side is always consuming the other, aiming to increase, as you can see in the Figure 2 [7].

In TCM terms, Blood functions are to nourish and vitalize the whole body. To maintain the Blood's free flowing state another energy force must work in harmony with it and this energy is $Q i$. Qi is the vital energy that invigorates Blood to keep it circulating throughout the body. Blood and $Q i$ have a very important relationship and are mutually dependent. Qi prevents Blood stagnation and Blood nourishes $Q i$. The aim of all treatments is to achieve a balanced state between Yin, Yang, Qi and Blood to achieve health, as you can see in the Figure 3 [7].

When there is an energy deficiency between one and a combination of these four energies, there is the formation of internal Heat, as you can see in the Figure 4. It can also be caused by incorrect diet, such

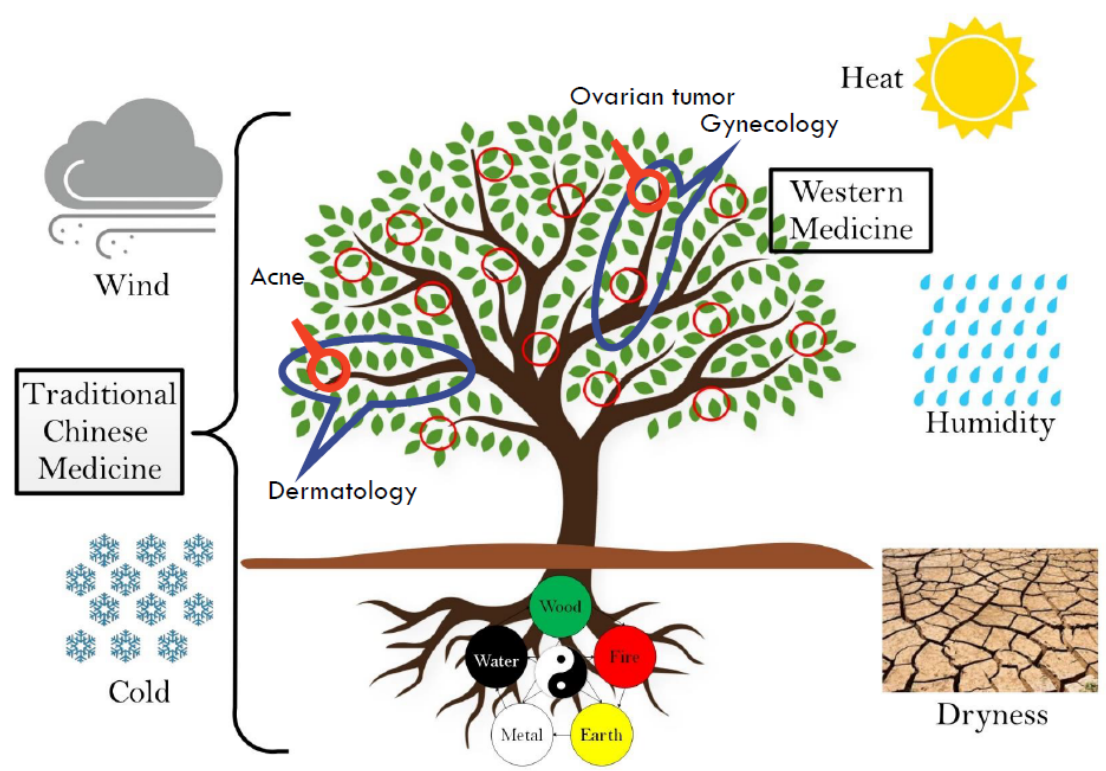

Figure 1: Tree metaphor of Western and traditional Chinese medicine. 


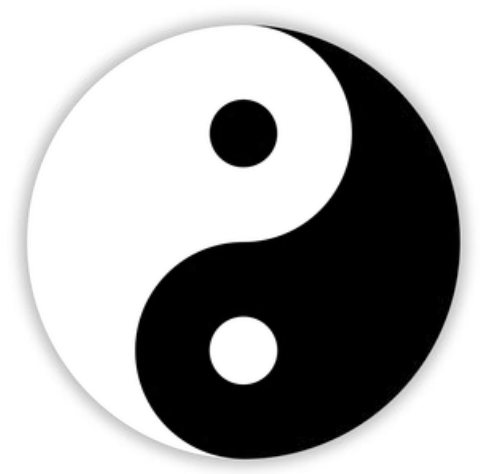

Figure 2: Yin and Yang symbol.

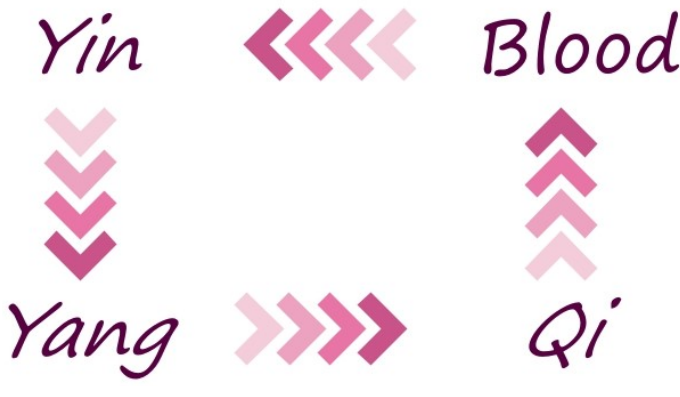

Figure 3: The Yin, Yang, Qi and Blood schematic relationship.

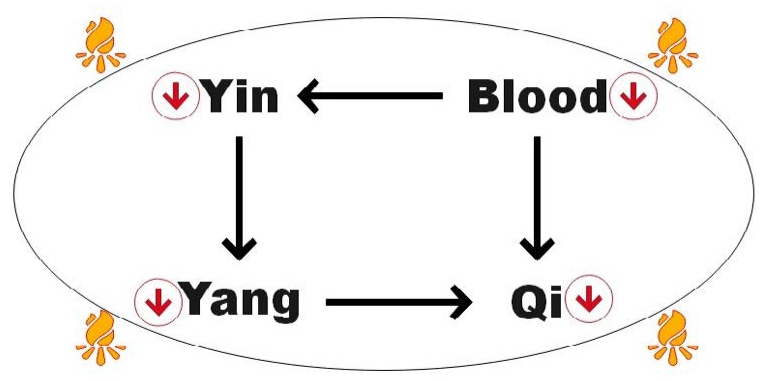

Figure 4: Internal Heat formation when there is energy deficiency.

as the constant consumption of fried foods, melted cheese, eggs, chocolate, coconut, honey and alcoholic beverages. The second reason for the increase production of internal Heat could be the emotional factors such as excessive anger. The internal Heat is one of the energy imbalances that is necessary to produce cancer, according to traditional Chinese medicine and written by me (2020) in the article entitled The Importance of Treating Energy Imbalances and Chakras Replenishment for Prevention and Treatment of Cancer. In this article, I am showing the importance of treating the energy deficiency state of three cases reports, the first two patients have diagnosis of thyroid cancer and pap smear alteration grade IV respectively, and were cured only doing energy rebalancing and replenishing the chakras' energy centers that were deficient in energy in both patients. And in the third case report, it was a male patient with Lung cancer diagnosis and only improved his metastasis condition, disappearing them when treating his energy deficiencies using the tools recommended in this article such as Chinese dietary counseling, auricular acupuncture with apex ear bloodletting and the use of highly diluted medications to improve the vital energy of the patient that was already low that was worsening with the use of chemotherapy or radiotherapy. When this third case report was using only chemotherapy, his metastasis was reducing in size but always appearing in another site of the body $[3,7]$.

The second main theory basing traditional Chinese medicine is the Five Elements theory. The Five Elements theory states that there are five elements present in everything in the world, including our bodies. These elements are Water, Wood, Fire, Earth and Metal and inside the human body, these elements will be represented by five specific massive organs. These organs have extremely important functions to produce internal energy to allow adequate functioning of the human body. The Wood element corresponds to the Liver, the Fire element corresponds to the Heart, the Earth element corresponds to the Spleen, the Metal element corresponds to the Lungs, and the Water element corresponds to the Kidney, as you can see in the Figure 5 [8].

The chakras are energy concentrations that you cannot see by the naked eyes. There are seven chakras and each one is responsible for sending energy to one specific organ that they command. There are studies in the literature correlating chakras' with the Five Elements theory in traditional Chinese medicine such as the study wrote by Chase (2018) entitled The Geometry of Emotions: Using Chakra Acupuncture and 5-Phase Theory to Describe Personality Archetypes for Clinical Use, that this author is correlating the five elements with the chakras' energy centers and for this reason, when I measure the chakras' energy centers, I am measuring the five internal massive organs energy and I will know if the organ has energy or not to work and production of energy that each one is responsible, as showed in the Figure 6 [9].

There are seven main chakras present in the body and their relationship among themselves is extremely important for the body's health. As there are seven chakras and five elements, the seventh chakra (spiritual) is ruled by the first (Wood or Liver), and the sixth chakra (memory and concentration) is ruled by the second (Water or Kidney). The fifth chakra is ruled by Earth (Spleen-Pancreas) and it is responsible for Stomach, Spleen, Pancreas, Thyroid, Breasts. The fourth is ruled by Metal (Lung) and it is responsible for the skin, hair and sense of smell and distribution of energy. The third chakra is ruled by Fire or Heart and it is responsible for speech and communication and sleeping process. The second is ruled by Water (Kidney) and it is responsible for the youth, hearing, memory and concentration, teeth, bones, reproduction and sexual function. The first chakra is ruled by Wood or Liver and it is responsible for distribution of energy in the entire body. The energy alteration could be happening before beginning the hormone treatment and was harmed even more after the hormone to treat acne begins because hormones is considered highly concentrated medications and according to Arndt-Shultz Law, can reduce even more this vital energy, leading to have more propensity to have cancer formation [10].

The first step used by me in all my treatments was the changes in the dietary aspects of the patient, to promote an equilibrium between the Yin and Yang and also, the five elements of the Five Elements theory. The dietary changes were well explained in the article Why Are Diabetic Patients Still Having Hyperglycemia Despite Diet Regulation, Antiglycemic Medication and Insulin? [7]. 


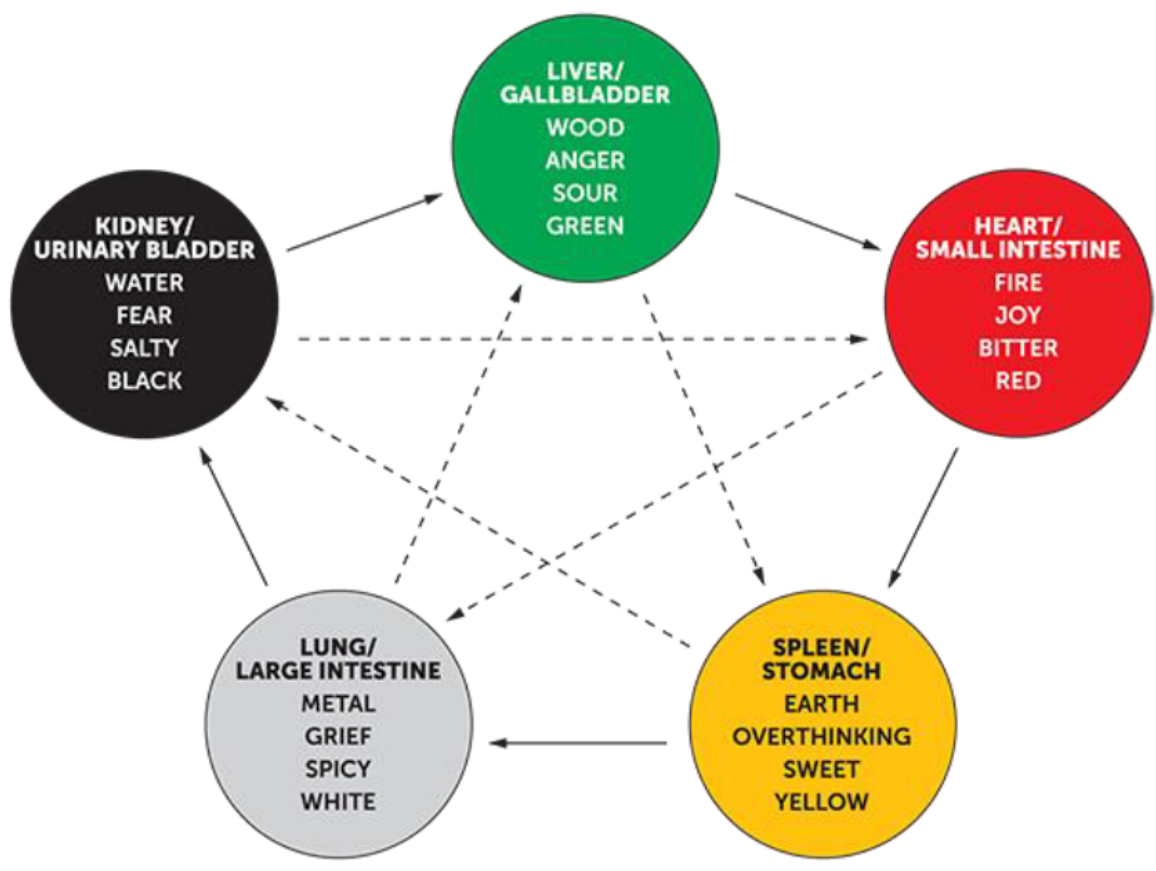

Figure 5: Five Elements theory.

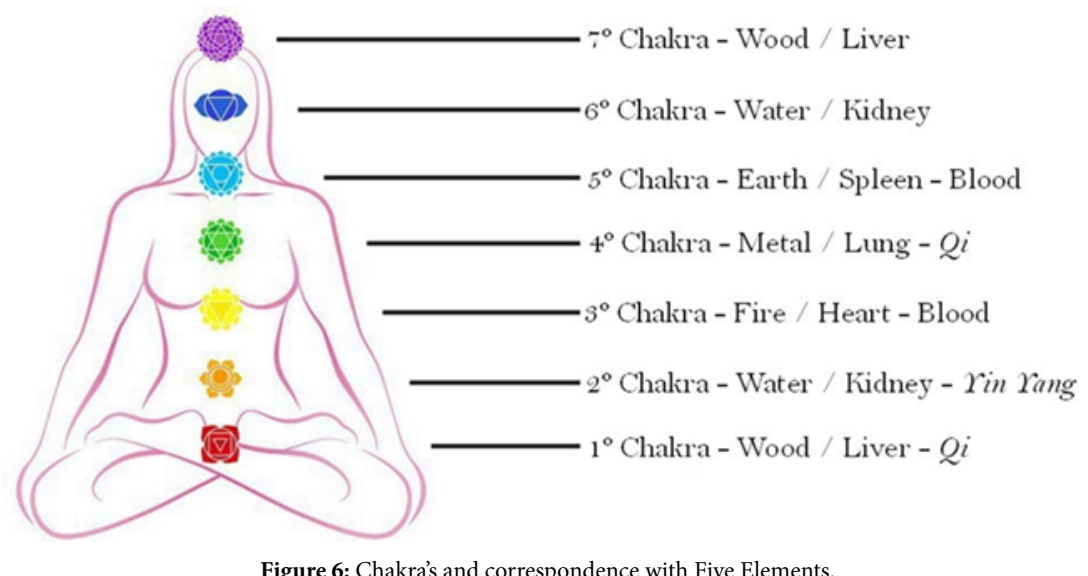

The second step in the treatment were the use of acupuncture associating with apex ear bloodletting, because is a very important tool used by me to regulate the Yin, Yang, Q $i$ and Blood, as I showed the meaning of each auricular point used in the treatment of this patient in the article entitled How Do You Treat Back Pain in Your Practice? [10].

Moxibustion is another toll used by Chinese medicine to increase the vital energy of the patient and in the case of this patient that was in treatment of malignant ovary tumor using highly concentrated medications, her vital energy was reduced and the use of moxibustion in this case would benefit the patient, increasing her energy to fight against the malignant cell production [11].

After these concepts been briefly introduced, we can talk about the acne and mainly about ovarian tumor formation. According to TCM, acne is caused by internal Heat retention and invasion of Dampness. And cancer is formed by energies deficiencies and Heat retention. Both studies about these subjects were written by me (2020) in the article entitled Energies Imbalances and Chakras' Energies Deficiencies in the Treatment of Acne and in the second article entitled The Importance of Treating Energy Imbalances and Chakras Replenishment for Prevention and Treatment Chakras Replenishment for Prevention and Treatment of Cancer among many others $[3,12]$.

What I am saying in all these articles about cancer formation is that they all have in the back ground, energy deficiencies in the chakras' energy centers and the treatment replenishing these energy is very important to improve the immune system of them allowing the body to fight against the production of malignant cell, that usually occurs every day, according to the study written by me (2020) entitled The Importance of Treating Energy Imbalances and Chakras Replenishment for Prevention and Treatment of Cancer [3].

In the article written by Cooper (2000) entitled The Development and Causes of Cancer, he is saying that the increase incidence of cancer with age suggests that cancer comes from the development of multiples abnormalities that accumulates over many years [13]. 
In this case reported in this article, the patient was using hormones for more than 10 years to treat acne and as I showed in the article (2020) entitled Energies Imbalances and Chakras' Energies Deficiencies in the Treatment of Acne, patients with acne also have chakras' energy centers deficient in energy and when introducing hormones to treat acne, the hormones is considered highly concentrated medications, that according to Arndt-Shultz Law ( Figure 7), can reduce the vital energy of the patient that was already low, leading to increase chance of having cancer in the future $[12,14]$.

The reason why the use of this kind of medication can increase the chance of having cancer in the future is explained by me in the Table 1. In this table, I am showing that the evolution from health to disease is divided in five phases, where the first three phases are characterized by the energy alterations (less energy) but the laboratorial exams are normal. In the phase four, the patient has symptoms and the laboratorial exams have some alterations but the phase five is characterized by the irreversible lesion caused by cancer formation. In the case of the patient reported in this article, when she began to have acne, probably that her energy was low and when it was prescribed the use of hormones to treat this acne condition, the energy dropped even more, reducing the vital energy that was important to fight against the formation of malignant cells, developing cancer after 10 years of using this kind of medication [3].

In the article written by me (2021) entitled What Are the Markers That Predict the Development of Having Cancer in the Future Without Laboratory or Radiological Tests? I am saying that the measurement of energy in the chakras' energy centers is a very important toll nowadays, to predict the evolution of the patient to cancer in the future because, what Western medicine is doing nowadays, is to do laboratory or radiological exams to do the diagnosis of cancer in the beginning of cancer formation. In my opinion, this kind of exam is not preventing the cancer formation but only doing cancer diagnosis in the early phase of the formation of cancer [15].

In the article I wrote (2021) entitled Energy Alterations and Chakras' Energy Deficiencies and Propensity to SARS-CoV-2 Infection, I did a research measuring the energy of 1000 patients during 2015 to 2020 and what I found was that $90 \%$ of all my patients, including babies, children, adolescents, young adults and older people, are in the lowest level of energy in all internal massive organs, as I am showing in the Table 2. In this study, I am demonstrating the majority of the population nowadays could be high risks to have cancer in some near future because energy is important to prevent the formation of cancer that usually grows when there is energy deficiency for long time [16].

The aim to treat the energy of this patient reported in this article was to increase the vital energy that was already low, even receiving the chemotherapy and radiotherapy, because she was doing a localized treatment for the malignant ovary tumor, but the energy deficiency that had in the back ground and cannot see by the naked eyes was not treated yet. For this reason, I used other tools to increase her immunity (energy) to become stronger and allowing her body to fight against malignant cell formation even in use of chemotherapy [3].

Patients with cancer that it is in use of chemotherapy or radiotherapy, should be treated using medications to increase the vital energy such as the homeopathies according to the theory Constitutional Homeopathy of the Five Elements based on Traditional Chinese Medicine, showed in the Table 3. The medications need to be used according to the results made by radiesthesia procedure and the given according to the Generation cycle, as you can see in the Figure 8 [8].

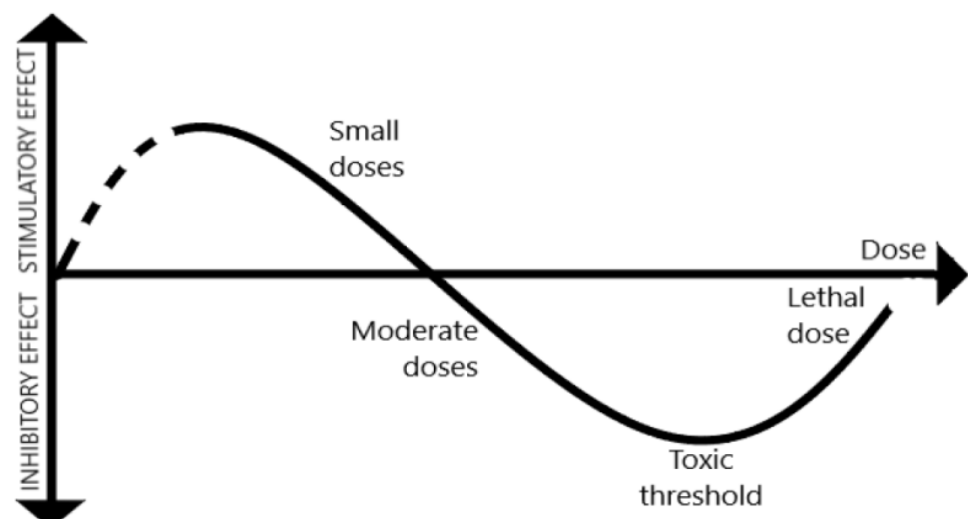

Figure 7: Arndt-Schultz Law.

Table 1: Evolution from health to disease formation.

\begin{tabular}{|l|c|c|c|}
\hline Phase & Organ & Exams & Energy Reserve \\
\hline 1 & Slowing down of organ functions & Normal & Normal \\
\hline 2 & Slowing down of organ functions & Normal & Consumption of internal energy reserves \\
\hline 3 & Slowing down of organ functions & Normal & Consumption of external energy reserves \\
\hline 4 & Reversible cellular lesion & Wittle alterations & Consumption of blood reserves \\
\hline 5 & Irreversible cellular lesion & Excessive alteration & Metabolic Exhaustion \\
\hline
\end{tabular}


Table 2: Research doing the chakras' energy centers measurement of 1000 patients in Brazil.

\begin{tabular}{|l|c|c|c|}
\hline Ages Chakras & $\mathbf{2 - 1 9}$ & $\mathbf{2 0 - 5 9}$ & $\mathbf{6 0 - 7 9}$ \\
\hline 7 & 8 & 8 & 8 \\
\hline 6 & 1 & 1 & 1 \\
\hline 5 & 1 & 1 & 1 \\
\hline 4 & 1 & 1 & 1 \\
\hline 3 & 1 & 1 & 1 \\
\hline 2 & 1 & 1 & 1 \\
\hline \multirow{2}{*}{1} & 1 & 1 & 86 \\
\hline \multirow{2}{*}{ Total of Patients } & 26 & 170 & Anxiety \\
\hline \multirow{3}{*}{ Main Western diagnoses } & Anxiety & Anxiety & Knee Pain \\
\cline { 2 - 5 } & Depression & Headache & Yin \\
\hline \multirow{3}{*}{ Main Chinese diagnoses } & Yin/Yang & Yin & Yin/Internal Heart \\
\cline { 2 - 5 } & & Yin/Yang & Yin/Yang/Internal Heart \\
\cline { 2 - 5 } & & &
\end{tabular}

Table 3: Homeopathy medications used in the treatment of lack of energy in the internal five massive organs.

\begin{tabular}{|l|c|c|c|}
\hline Chakras & Five Elements & Homeopathy Medications & Crystal-based medications \\
\hline $1^{\circ}$ Chakra & Wood/Liver & Phosphorus & Garnet \\
\hline $2^{\circ}$ Chakra & Water/Kidney & Natrum muriaticum & Orange calcite \\
\hline $3^{\circ}$ Chakra & Fire/Heart & Sulphur & Rhodochrosite \\
\hline $4^{\circ}$ Chakra & Metal/Lung & Silicea & Emerald \\
\hline $5^{\circ}$ Chakra & Earth/Spleen & Calcarea Carbonica & Blue Quartz \\
\hline $6^{\circ}$ Chakra & Water/Kidney & Tone $2^{\circ}$ Chakra & Sodalite \\
\hline $7^{\circ}$ Chakra & Water/Liver & Tone $1^{\circ}$ Chakra & Tiger eye \\
\hline
\end{tabular}

The homeopathy medications are recommended to use for one year or more (I think that if the high authorities do not have any action to see other possible ways to have modern technology of telecommunication, the use of this kinds of medications should be for the entire life of the patients), because of the influences of the $5 \mathrm{G}$ technology, leading to reduction of this vital energy that is low in quite the majority of the people in this world. I am doing a correlation between what I found in my patients in Brazil because the cause of this reduction in the energy in the five internal massive organs is caused by the electromagnetic radiation, that all people in this globe is suffering every day [16,17].

In this patient reported in this article, she had relapse of her malignant ovary tumor, when stopping the energy based treatment when begin the COVID-19 pandemic. This is to show the importance to strength the vital energy to prevent the formation of metastasis of this patient and other complications from the use of highly concentrated medications to treat the cancer itself [3].

In another article written by me (2021) entitled is there a Greater Risk in the Use of Hormones Nowadays? I am saying that the use of hormones by women should be made with precautions because of this new energy pattern of the population, that was different from people before 2015, when I am saying that patient have energy at that time, as I wrote (2021) in the article entitled Is the Population in the World the Same as in the Past? [18,19].

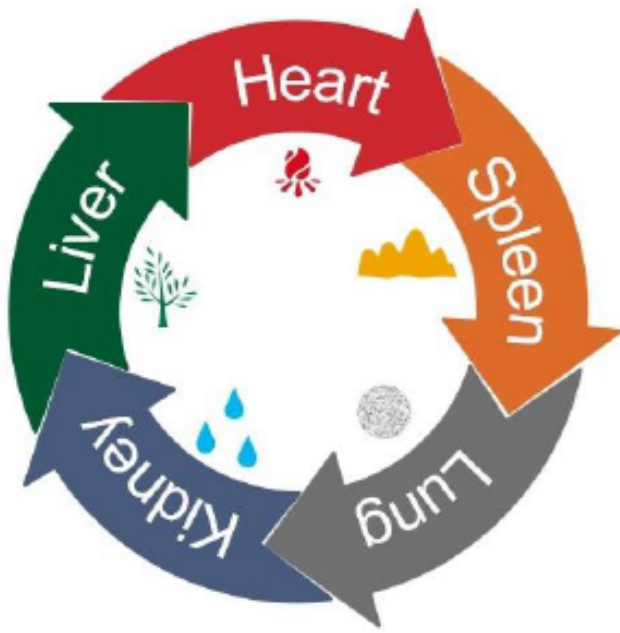

Figure 8: Generation Cycle of the Five Elements theory.

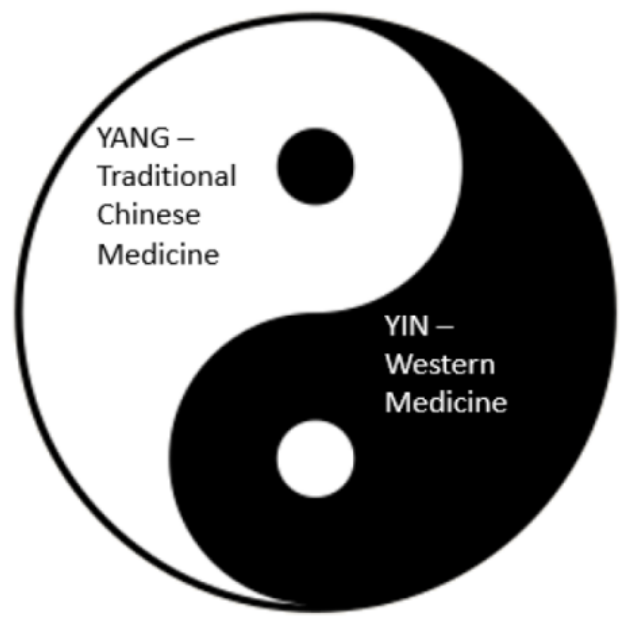

Figure 9: Yin and Yang of Western and traditional Chinese medicine metaphor.

I would like to emphasize that Acupuncture and Homeopathy are considered medical specialties since 1995 and 1980, respectively by the Federal Medical Council in Brazil [20].

That is why it is important to emphasize the importance of increasingly integrating Western and traditional Chinese medicine, as in this metaphor with the symbol of Yin and Yang one representing the Western medicine (Yin energy) that is materialized energy and traditional Chinese medicine represents Yang energy that is nonmaterialized energy, as you can see in the Figure 9. Both medicines can work together to understand better how disease is formed in the deepest level, and the importance of treating the leaf level but also, the root level of the tree, showed in the Figures 1 and 2 [7].

\section{Conclusion}

The conclusion of this study is that the use in the hormones can induce the formation of malignant ovary tumor in person with chakras' energy centers deficient in energy. The correction of the lack of energy (diagnosed by radiesthesia procedure) is a very important tool nowadays, to reduce the chance of having any chronic diseases and reduce of evolution to any type of cancer formation, in this 
case, malignant ovary cancer. The use of Chinese dietary counseling, auricular acupuncture with apex ear bloodletting and use of highly diluted medications according to Constitutional Homeopathy of the Five Elements based in Traditional Chinese Medicine and crystal-based medications, are very important step in the treatment of this kinds of diseases because can prevent complications from the use of treatment in Western medicine and also, reducing the chance of having relapses or even the formation of new type of cancer, if this patient still do not treat the energy deficiency state.

\section{References}

1. Momenimovahed Z, Tiznobaik A, Taheri S, Salehiniya H (2019) Ovarian cancer in the world: epidemiology and risk factors. Int J Womens Health 11: 287-299. [crossref]

2. Craik E (2014) The "Hippocratic" Corpus: Content and Context. Routledge 344.

3. Huang Wei Ling (2020) The Importance of Treating Energy Imbalances and Chakras Replenishment for Prevention and Treatment Chakras Replenishment for Prevention and Treatment of Cancer. Advances in Cancer Research \& Clinical Imaging 3.

4. Ho SM (2003) Estrogen, progesterone and epithelial ovarian cancer. Reprod Biol Endocrinol 1: 73-80. [crossref]

5. Bagatin E, Freitas THP De, Rivitti-Machado MC, Ribeiro BM, Nunes S, et al. (2019) Adult female acne: a guide to clinical practice. An Bras Dermatol 94: 62-75. [crossref]

6. Huang Wei Ling (2019) The Importance of Correcting Energy Imbalances and Chakras Energy Deficiencies in the Treatment of Patients with Glaucoma. Clin Res Ophthalmol 2: 1-9.

7. Huang Wei Ling (2019) Why Are Diabetic Patients Still Having Hyperglycemia despite Diet Regulation, Antiglycemic Medication and Insulin? Int J Diabetes Metab Disord 4: 1-14.

8. Huang Wei Ling (2020) Constitutional Homeopathy of Five Elements Based on Traditional Chinese Medicine. Acta Scientific Medical Sciences 4: 57-69.

9. Chase CR (2018) "The Geometry of Emotions: Using Chakra Acupuncture and 5-Phase Theory to Describe Personality Archetypes for Clinical Use". Medical Acupuncture 30: 167-178. [crossref]

10. Huang Wei Ling (2018) "How Do You Treat Back Pain in Your Practice? Part 2", Medical Acupunture 30: 46-53. [crossref]

11. Deng H, Shen X (2013) The Mechanism of Moxibustion: Ancient Theory and Modern Research. Evid Based Complement Alternat Med 2013: 379291. [crossref]

12. Huang WL (2020) Energies Imbalances and Chakras' Energies Deficiencies in the Treatment of Acne. Clinic Res Dermatol 3: 1-9.

13. Cooper GM (2000) The Development and Causes of Cancer. Sunderland (MA): Sinauer Associates.

14. Arndt Schultz Law and its applications in Homeopathy | Homeopathy Resource by Homeobook.com [Internet]. [cited 2020 Aug 5].

15. Huang WL. What Are the Markers That Predict the Development of Having Cancer in the Future Without Laboratory or Radiological Tests? Journal of Cancer and Cancer Prevention 1.

16. Huang Wei Ling (2021) "Energy Alterations and Chakras' Energy Deficiencies and Propensity to SARS-CoV-2 Infection”. Acta Scientific Microbiology 4.4: 167-196.

17. Huang WL (2021) The Influence of Cell Phones and Computers on Our Immune System. Ann Immunol Immunother 3: 000141.

18. Huang Wei Ling (2021) "Is there a Greater Risk in the Use of Hormones Nowadays?" Acta Scientific Women's Health 3.8: 08-10.

19. Huang Wei Ling (2021) "Is the Population in the World the Same as in the Past?". Acta Scientific Clinical Case Reports 2: 6.

20. Homeopatia e acupuntura reconhecidas e procuradas - Cremepe [Internet]. [cited 2020 Dec 9].

\section{Citation:}

Huang WL (2021) Energy Deficiency Leading to Predisposition of Formation of Malignant Ovary Tumor Secondary to Chronic Use of Hormones to Treat Acne. Integr Gyn Obstet J Volume 4(3): 1-8. 